\title{
Street Rehab: Linking Accessibility and Rehabilitation*
}

\author{
Catherine Holloway, Member, IEEE, Behzad Heravi, Member, IEEE, Giulia Barbareschi, Member, \\ IEEE, Sarah Nicholson, Stephen Hailes
}

\begin{abstract}
As part of the Accessible Routes from Crowdsourced Cloud Services project (ARCCS) we conducted a series of experiments using the ARCCS sensor to identify push style of wheelchair users. The aim of ARCCS is to make use of a set of well-calibrated sensors to establish a processing chain that then provides ground truth of known accuracy about location, the nature of the environment, and physiological effort. In this paper we focus on two classification problems 1) The push style employed by people as they push themselves and 2) Whether the person is being pushed by an attendant or pushing themselves (independent of push style). Solving the first enables us to develop a level of granularity to pushing classification which transcends rehabilitation and accessibility. The first problem was solved using a wrist-mounted ARCCS sensor, and the second using a wheel-mounted ARCCS sensor. Push styles were classified between semi-circular and arc styles in both indoor and outdoor environments with a high-decrees of precision and recall $(>95 \%)$. The ARCCS sensor also proved capable of discerning attendant from self-propulsion with near perfect accuracy and recall, without the need for a body-worn sensor.
\end{abstract}

\section{INTRODUCTION}

In high-income countries, a leading cause of death is inactivity. In the UK it is the $4^{\text {th }}$ largest cause of disability and death [1], making it as deadly as smoking [2]. Manual wheelchair users have been shown to be less prone to meet health guidelines for physical activity, which puts them at greater risk for cardiovascular, musculoskeletal and metabolic disorders [3], [4][5]. The associated increase in body mass might further favor the likelihood of developing upper limb pain and injury, causing further damage to the individual's health and social participation [6], [7]. A proven method for successfully increasing activity levels is to embed active travel into a daily commute to work or school. However, wheelchair users frequently encounter barriers in the built environment, which prevent them from being able to embed active travel into their daily routine. In order to build such activity patterns into a routine, there are two issues to consider - the basic need of accessibility (i.e. can the person physically push themselves to the place) and injury prevention (i.e. will the route invoke a push style and pattern which might, in the long-term, cause upper-limb injury). Approximately half of the manual wheelchair user population will suffer an upper-limb injury [8], [9]. Injury prevalence has been linked to age and time spent in a wheelchair [10] and push styles which create high peak forces with a rapid rate of rise of force [11]. Such forces have been shown to be linked to the environment [12]. According to the Guidelines for Preservation of Upper Limb Function Following SCI, a semicircular push style should be encouraged during rehabilitation and wheelchair training as it reduces the likelihood of pain development [13]. However, pushing style adopted outdoor is affected by environmental conditions with people needing to adapt their push-style when pushing up hills [14]. Therefore there is a need to link both accessibility and rehabilitation parameters.

The aim of the ARCCS project is to make use of a set of well-calibrated sensors to establish a processing chain that then provides ground truth of known accuracy about location, the nature of the environment, and physiological effort. In doing so we are enabling the wheelchair to become a part of the Internet of Things. In previous work we have investigated the types of sensors [15] which could be used for such a system and shown measurements obtained from both smartphones and standalone Inertial Measuring Units (IMUs) sensor devices can be used with a high level of credibility in wheelchair accessibility studies. By crowd-sourcing such data ARCCS will allow wheelchair users with similar self-identified levels of ability to use each other's suggested routes and times in order to plan their journey. ARCCS combines and advances work which has been completed in the activity monitoring/rehabilitation and geomatic information services domains.

Here we focus on how ARCCS can advance the rehabilitation and activity monitoring domains. A number of studies have investigated the use of wheel mounted accelerometers to measure the activity of wheelchair users and have found good correlations $(>0.99 \mathrm{IC}(2,1))$ from this data to number of wheel rotations and duration of movement. Learmonth and colleagues [16] have recently established cutoff points in the vector magnitude of accelerations (VMA) measured using a wrist-worn triaxial AciGraph accelerometer system. The study took place on a treadmill across a number of speeds and with 24 wheelchair users. It established cut-off points for $1.5 \mathrm{mph}(2.4 \mathrm{kmph}), 3.0 \mathrm{mph}(4.8 \mathrm{kmph})$ and $4.5 \mathrm{mph}$ (7.2 kmph). Further, it demonstrated a strong linear correlation between the VMA and VO2, thus allowing a method for characterizing physical activity of wheelchair users. The study is limited, as admitted by the authors, is that the focus was on physically active wheelchair users who were capable of propelling $4.5 \mathrm{mph}$ for $6 \mathrm{~min}$. Many wheelchair users do not achieve such exercise bouts. Sonenblum et al. [17] has shown the median activity bout (of 28 manual wheelchair users) lasts $21 \mathrm{~s}$, resulting in $8.1 \mathrm{~m}$ travelled and a velocity of $1.5 \mathrm{kmph}$. [18] have used a wrist-mounted accelerometer to classify the push style with an accuracy of over $90 \%$ using a k-Nearest Neighbor $(\mathrm{kNN})$ classifier.

More recently [19] claim that the draw-back of a single device is that it can only be mounted in one place. If wheelmounted it is unable to measure upper-limb movement, and if on the arm it is incapable of measuring wheelchair movements A particular criticism of wheel-mounted sensors is that they are unable to distinguish between self-propulsion and attendant propulsion [19]. The researchers therefore use a gyro based wheel sensor combined with an accelerometer based 
system placed on the arm (both wrist and upper arm are trialed). This system allows for different activities to be identified, using a two-step process. The process first identifies the stationary, near-stationary or moving and then further classifies an activity (e.g. folding clothes).

\section{METHODS}

The aim of this study was to expand the work done by [18] by introducing self-selected speed and therefore a variety of velocities and to establish classifiers which would therefore be able to detect push styles both indoors and outdoors over a variety of terrains. In addition, we wished to investigate if it was possible to detect attendant-pushing from self-pushing using a wheel-mounted sensor to help determine the best placement of ARCCS sensors for future studies. The wheelbased sensor would be attractive if we could identify between attendant and self propulsion as it would allow us to discern how much activity a person was getting from an unobtrusive sensor.

\section{A. The ARCCS sensor}

The sensor node developed in ARCSS (Figure 2) uses a system-on-a-chip processor that has embedded wireless capabilities. It contains a 3D accelerometer, 3D gyroscope, 3D magnetometer, an absolute pressure sensor (to detect when the person is going uphill), a micro SD card and Bluetooth transmission capabilities. It also contains a realtime clock, used to wake the device at particular times to conserve battery. A bespoke 3D printed bracelet and wheel mount were created for the sensor and used for data collection. The sensor and mounts are shown in Figure 1.

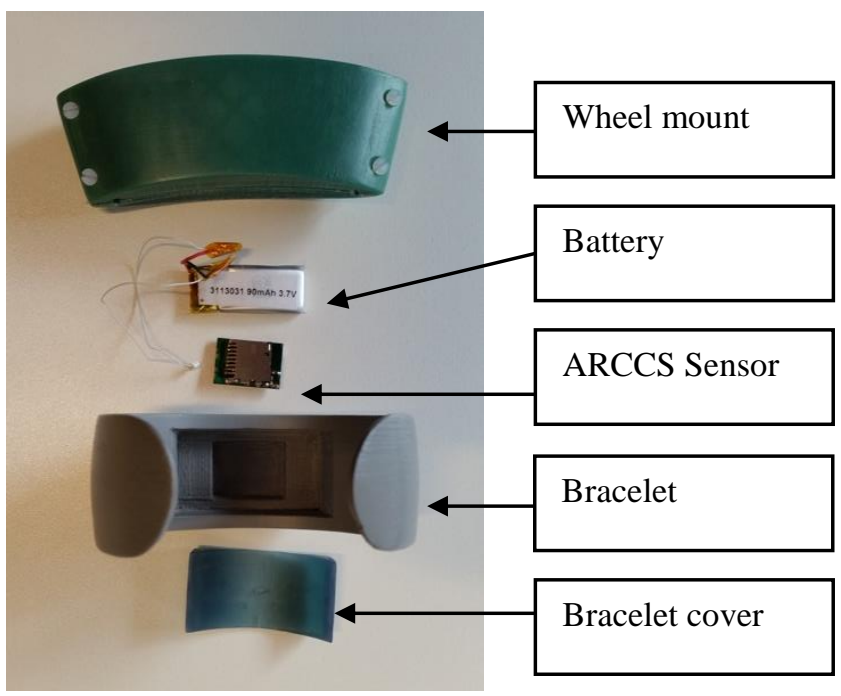

Figure 1: ARCCS sensor, battery and mounts.

\section{A. Data collection}

Initial data was collected along a $10 \mathrm{~m}$ corridor with low pile carpet. Non-regular wheelchair users were asked to push along the corridor, turn and return and to complete this until they had completed 5 'loops' of the corridor. They first completed this using a semi-circle push style and then using an arc push style. Data was collected by a single participant who was not a regular wheelchair user, but who learnt the push styles in the presence of a physiotherapist over several days prior to data collection. A follow-up data set was completed over different terrains outside, these terrains formed a loop around a block of the University campus; again the subject completed the loop first with a semi-circle style and then with an arc style. Data was recorded at $100 \mathrm{~Hz}$.

\section{B. Data Processing}

Data was initially visualized in the ARCCS data visualizer to check for any errors in the data and to confirm the start and end time of each trial. The data was then pre-processed in Matlab (Version R2015a). The orientation of the sensor was calculated using the three channels of accelerometer and three channels of gyroscope data as inputs for the Madgwick filter [20]. Time domain features were then extracted. These consisted of mean and standard deviation the 3 channels of acceleration, gyroscope and angle. An ARFF file was built in Matlab to be used in WEKA (V.3.16.13).

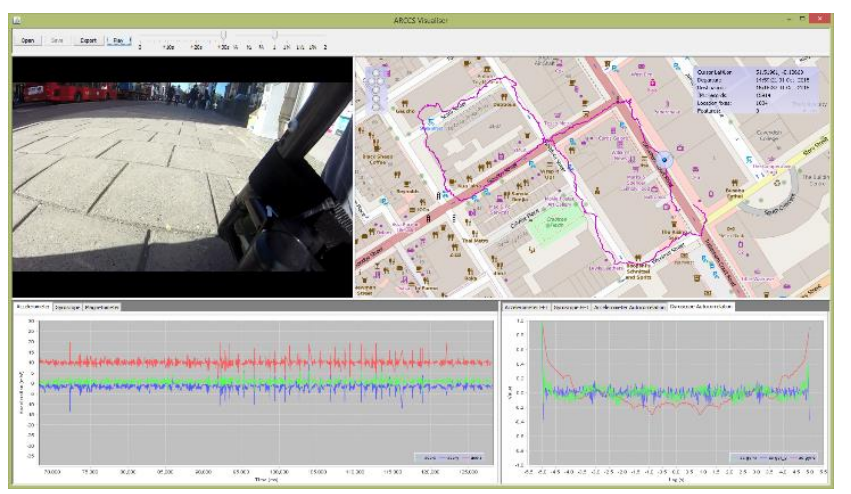

Figure 2: visARCCS, the ARCCS visualization tool

\section{Classification of push patterns}

Classifiers were trained in WEKA. A 10-fold-cross validation (10-fold-CV) was preformed using a naïve Bayesian classifier and a sequential minimal optimization algorithm (SMO) obtained by training a support vector machine (SVM) classifier.

\section{Push Styles}

Distributions of each of the attributes are shown in Figure 3.

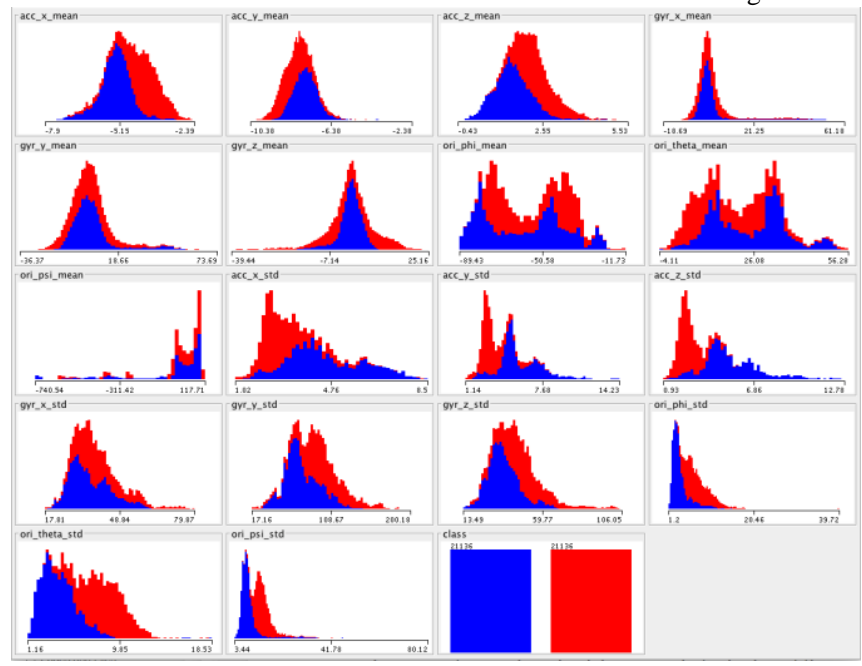

Figure 3: Pre-processed variable distributions in WEKA 
All 18 attributes were used in the classification and both NB and reliable results (see Table 1). The SVM classifier was more accurate over the NB classifier though both achieved good results.

Table 1: Results of NB and SVM classifiers on indoor and outdoor wrist-mounted sensor data

\begin{tabular}{|l|l|l|l|l|}
\hline & $\begin{array}{l}\text { True } \\
\text { positive }\end{array}$ & $\begin{array}{l}\text { False } \\
\text { positive }\end{array}$ & Precision & Recall \\
\hline $\begin{array}{l}\text { Indoor } \\
\text { (NB) }\end{array}$ & .954 & .008 & .991 & .954 \\
\hline $\begin{array}{l}\text { Indoor } \\
\text { (SVM) }\end{array}$ & .997 & .003 & .997 & .997 \\
\hline $\begin{array}{l}\text { Outdoor } \\
\text { (NB) }\end{array}$ & .986 & 0 & 1 & .986 \\
\hline $\begin{array}{l}\text { Outdoor } \\
\text { (SVM) }\end{array}$ & .998 & 0 & 1 & .998 \\
\hline
\end{tabular}

The confusion matrices for both indoor and outdoor data are given in Table 2. For both classifiers it was more likely that the SVM gave more accurate classifications.

Table 2: Confusion matrices from the NB and SVM classifiers on indoor and outdoor wrist-mounted sensor data with predicted labels given in each column.

\begin{tabular}{|l|l|l|l|l|l|}
\cline { 3 - 6 } \multicolumn{2}{c|}{} & NB & NB & SVM & SVM \\
\cline { 3 - 6 } \multicolumn{2}{c|}{} & Arc & Semi & Arc & Semi \\
\hline $\begin{array}{l}\text { True } \\
\text { labels } \\
\text { (indoor) }\end{array}$ & Arc & 20161 & 975 & 21079 & 57 \\
\cline { 2 - 6 } & $\begin{array}{l}\text { Semi- } \\
\text { circle }\end{array}$ & 174 & 20962 & 71 & 21065 \\
\hline $\begin{array}{l}\text { True } \\
\text { labels } \\
\text { (Outdoor) }\end{array}$ & Arc & 10998 & 153 & 11130 & 21 \\
\cline { 2 - 6 } & $\begin{array}{l}\text { Semi- } \\
\text { circle }\end{array}$ & 0 & 11151 & 0 & 11151 \\
\hline
\end{tabular}

\section{E. Attendant V's Pushed from wheel sensor}

Precision values were $100 \%$ on this data set when an SVM or NB classifier was used, with recall recorded as $100 \%$ and $96.1 \%$ respectively. This high level of correct classification fell when attributes from only the accelerometer were used (mean and standard deviations from each channel). Precision dropped to $75 \%$ with the NB and $82 \%$ with the SVM; recall was $73 \%$ for the NB and $71 \%$ for SVM. These lower levels are reflected in the confusion matrices shown in Table $3 \& 4$.

Table 3: Confusion matrices self-propelled (self) and attendantpropelled (attendant) with gyro and accelerometer-based attributes.

\begin{tabular}{|c|c|c|c|c|c|}
\hline & \multicolumn{4}{|c|}{$\begin{array}{l}\text { Predicted labels - Acceleration and } \\
\text { orientation attributes }\end{array}$} \\
\hline & & $\begin{array}{l}\text { Attendant } \\
\text { NB }\end{array}$ & $\begin{array}{l}\text { Self } \\
\text { NB }\end{array}$ & $\begin{array}{l}\text { Attendant } \\
\text { SVM }\end{array}$ & $\begin{array}{l}\text { Self } \\
\text { SVM }\end{array}$ \\
\hline \multirow{2}{*}{ 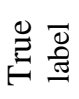 } & Attendant & 770 & 31 & 801 & 0 \\
\hline & Self & 0 & 801 & 0 & 801 \\
\hline
\end{tabular}

Table 4:Confusion matrices self-propelled (self) and attendantpropelled (attendant) with accelerometer-based attributes.

\begin{tabular}{|c|c|c|c|c|c|}
\hline & \multicolumn{4}{|c|}{$\begin{array}{l}\text { Predicted labels - Acceleration and } \\
\text { orientation attributes }\end{array}$} \\
\hline & & $\begin{array}{l}\text { Attendant } \\
\text { NB }\end{array}$ & $\begin{array}{l}\text { Self } \\
\text { NB }\end{array}$ & $\begin{array}{l}\text { Attendant } \\
\text { SVM }\end{array}$ & $\begin{array}{l}\text { Self } \\
\text { SVM }\end{array}$ \\
\hline \multirow{2}{*}{ 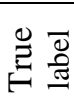 } & Attendant & 585 & 216 & 572 & 229 \\
\hline & Self & 193 & 608 & 127 & 674 \\
\hline
\end{tabular}

\section{DISCUSSION}

The results presented in this study have demonstrated the ability to classify push styles using a wrist-mounted IMU using only time-based attributes. Our results, using selfselected speeds, have shown similar accuracy in classifications as [21], which is the nearest research to that we present. This has been accomplished with the addition of gyroscope and angle attributes, and has meant we did not need to use frequency-based attributes which have been needed in previous studies (e.g. [19]). The use of lesscomputationally demanding attributes has the potential to enable us to process and classify pushes onboard the ARCCS sensor before streaming simple metrics of a journey or exercise bout to a wheelchair user's mobile phone or tablet. In keeping with results from [18] we also found that when the rolling resistances were higher (i.e. outdoors) the classifiers were more accurate.

We believe our study complements existing literature which has focused on classifying distinct activities (e.g. basketball, from household chores and regular pushing [19]). and on studies which have validated wheel-mounted accelerometerbased methods for measuring wheelchair activity bouts (e.g. [22]). In our study we add a level of granularity to wheelchair pushing classification moving from activities to the quality of movement within an activity, which builds on the initial work of [21]. This level of granularity opens up the possibility of further investigation into how environmental conditions affect pushing styles and to monitor how well people learn to apply pushing styles during wheelchair skills training in real life conditions. This paper presents what the authors believe to be the potential to realize the ARCCS system. However, data has been collected and cross-validated on a single subject, and it is entirely possible that performance might drop when applying the classifier to another person. Future work should address this issue by collecting a larger dataset. In addition, the results of this granular-level push-style classification and the identification of hands on and off timings should be combined. These could then be used to constrain a calculated path to that of the wheel between these points The second aim of this paper was to demonstrate the feasibility of identifying when a person is being pushed by an attendant and when they are self-propelling using only a wheel-mounted sensor. Our results show that when only accelerometer-based attributes are used the accuracy of the classifiers drops to $75 \%$ with a NB classifier and $82 \%$ with 
an SMO algorithm. However, when the gyro-based attributes were included the accuracy increased to $100 \%$. It must be stressed that while a range of velocities were used for both pushing and pushed the sample was still quite small. This is also true for the self-propelled study. In addition, we were unable to test sensors on both the wheel and the wrist simultaneously as we had only manufactured a single sensor. Therefore, future work will focus on collecting a larger data set, with wheelchair users across a longer time period.

\section{CONCLUSION}

This study shows the potential of using a wrist worn IMU to detect pushing styles across different surfaces and at various self-selected speed. In addition, it shows it is possible to use a single sensor mounted on the wheel to distinguish between self and attendant wheelchair pushing. These results advance the possibilities for linking wheelchair rehabilitation and accessibility measures in the future; and make possible the identification of pushing styles which are more likely to cause injury as a person pushes every day.

\section{REFERENCES}

[1] C. J. Murray, M. A. Richards, J. N. Newton, K. A. Fenton, H. R. Anderson, C. Atkinson, D. Bennett, E. Bernabé, H. Blencowe, R. Bourne, T. Braithwaite, C. Brayne, N. G. Bruce, T. S. Brugha, P. Burney, M. Dherani, H. Dolk, K. Edmond, M. Ezzati, A. D. Flaxman, T. D. Fleming, G. Freedman, D. Gunnell, R. J. Hay, S. J. Hutchings, S. L. Ohno, R. Lozano, R. A. Lyons, W. Marcenes, M. Naghavi, C. R. Newton, N. Pearce, D. Pope, L. Rushton, J. A. Salomon, K. Shibuya, T. Vos, H. Wang, H. C. Williams, A. D. Woolf, A. D. Lopez, and A. Davis, "UK health performance: findings of the Global Burden of Disease Study 2010," The Lancet, vol. 381, no. 9871, pp. 997-1020, Mar. 2013.

[2] C. P. Wen and X. Wu, "Stressing harms of physical inactivity to promote exercise," Lancet Lond. Engl., vol. 380, no. 9838, pp. 192193, Jul. 2012.

[3] S. Kinne, D. L. Patrick, and D. L. Doyle, "Prevalence of Secondary Conditions Among People With Disabilities," Am. J. Public Health, vol. 94, no. 3, pp. 443-445, Mar. 2004.

[4] C. A. Warms, J. D. Whitney, and B. Belza, "Measurement and description of physical activity in adult manual wheelchair users," Disabil. Health J., vol. 1, no. 4, pp. 236-244, Oct. 2008.

[5] Public Health England, "Everybody Active, Every Day. An evidencebased approach to physical activity," Sep. 2014.

[6] J. L. Collinger, M. L. Boninger, A. M. Koontz, R. Price, S. A. Sisto, M. L. Tolerico, and R. A. Cooper, "Shoulder Biomechanics During the Push Phase of Wheelchair Propulsion: A Multisite Study of Persons With Paraplegia," Arch. Phys. Med. Rehabil., vol. 89, no. 4, pp. 667-676, Apr. 2008.

[7] M. L. Boninger, R. A. Cooper, M. A. Baldwin, S. D. Shimada, and A. Koontz, "Wheelchair pushrim kinetics: Body weight and median nerve function," Arch. Phys. Med. Rehabil., vol. 80, no. 8, pp. 910915, Aug. 1999.

[8] I. H. Sie, R. L. Waters, R. H. Adkins, and H. Gellman, "Upper extremity pain in the postrehabilitation spinal cord injured patient," Arch. Phys. Med. Rehabil., vol. 73, no. 1, pp. 44-48, 1992.

[9] H. Gellman, D. R. Chandler, J. Petrasek, I. Sie, R. Adkins, and R. L. Waters, "Carpal tunnel syndrome in paraplegic patients," J. Bone Jt. Surg., vol. 70, no. 4, p. 517, 1988.

[10] M. Akbar, M. Brunner, G. Balean, T. Grieser, T. Bruckner, M. Loew, and P. Raiss, "A cross-sectional study of demographic and morphologic features of rotator cuff disease in paraplegic patients," $J$. Shoulder Elb. Surg. Am. Shoulder Elb. Surg. Al, vol. 20, no. 7, pp. 1108-1113, Oct. 2011.

[11] M. L. Boninger, B. G. Impink, R. A. Cooper, and A. M. Koontz, "Relation between median and ulnar nerve function and wrist kinematics during wheelchair propulsion," Arch. Phys. Med. Rehabil., vol. 85, no. 7, pp. 1141-1145, Jul. 2004.

[12] C. S. Holloway, A. Symonds, T. Suzuki, A. Gall, P. Smitham, and S. Taylor, "Linking wheelchair kinetics to glenohumeral joint demand during everyday accessibility activities," in 2015 37th Annual International Conference of the IEEE Engineering in Medicine and Biology Society (EMBC), 2015, pp. 2478-2481.

[13] "Preservation of Upper Limb Function Following Spinal Cord Injury," J. Spinal Cord Med., vol. 28, no. 5, pp. 434-470, 2005.

[14] W. M. Richter, R. Rodriguez, K. R. Woods, and P. W. Axelson, "Stroke Pattern and Handrim Biomechanics for Level and Uphill Wheelchair Propulsion at Self-Selected Speeds," Arch. Phys. Med. Rehabil., vol. 88, no. 1, pp. 81-87, Jan. 2007.

[15] M. Gupta, C. Holloway, B. M. Heravi, and S. Hailes, "A comparison between smartphone sensors and bespoke sensor devices for wheelchair accessibility studies," in 2015 IEEE Tenth International Conference on Intelligent Sensors, Sensor Networks and Information Processing (ISSNIP), 2015, pp. 1-6.

[16] Y. C. Learmonth, D. Kinnett-Hopkins, I. M. Rice, J. L. Dysterheft, and R. W. Motl, "Accelerometer output and its association with energy expenditure during manual wheelchair propulsion," Spinal Cord, vol. 54, no. 2, pp. 110-114, Feb. 2016.

[17] S. E. Sonenblum, S. Sprigle, and R. A. Lopez, "Manual Wheelchair Use: Bouts of Mobility in Everyday Life," Rehabil. Res. Pract., vol. 2012, pp. 1-7, 2012.

[18] B. French, A. Smailagic, D. Siewiorek, V. Ambur, and D. Tyamagundlu, "Classifying wheelchair propulsion patterns with a wrist mounted accelerometer," in Proceedings of the ICST 3rd international conference on Body area networks, ICST, Brussels, Belgium, Belgium, 2008, pp. 20:1-20:4.

[19] S. V. Hiremath, S. S. Intille, A. Kelleher, R. A. Cooper, and D. Ding, "Detection of physical activities using a physical activity monitor system for wheelchair users," Med. Eng. Phys., vol. 37, no. 1, pp. 68 76, Jan. 2015.

[20] Sebastian Madgwick, Open source IMU and AHRS algorithms. 2009.

[21] B. French, A. Smailagic, D. Siewiorek, V. Ambur, and D. Tyamagundlu, "Classifying wheelchair propulsion patterns with a wrist mounted accelerometer," in Proceedings of the ICST 3rd international conference on Body area networks, 2008, p. 20.

[22] S. E. Sonenblum, S. Sprigle, J. Caspall, and R. Lopez, "Validation of an accelerometer-based method to measure the use of manual wheelchairs," Med. Eng. Phys., vol. 34, no. 6, pp. 781-786, Jul. 2012. 